\title{
Reducción de oscilaciones en BitTorrent mediante mecanismos de unchoking preferencial
}

\section{Reduction of oscilations in BitTorrent by preferential unchoking mechanisms}

Resumen: Dado el crecimiento de los sistemas peer-to-peer y en particular del protocolo BitTorrent para la distribución de contenido en redes, han surgido modelos que buscan comprender su funcionamiento y predecir su desempeño. En este trabajo realizamos estudios de simulación en NS-2 para verificar la validez de uno de estos modelos, que predice la open siculo equilios acilas pueden conducir a fallos de funcionamiento. A partir questos resultados proponemos una variante del protocolo BitTorrent, tendiente a lograr mayor variante del protocolo Bit matematico, evaluamos las mejoras de ofrece esta variante. Redes peer-to-peer. BitTorrent. Simulaciones. Modelado. Algoritmos de choking.

Abstract: Given the growth of peer-to-peer systems and in particular the BitTorrent protocol for content distribution various models have been developed in order to understand its behavior and predict its performance. In this work its behavior and predict its performance. In this work that predicts operation around a stable equilibrium. We found situations where the model equilibrium predictions are adequate, but also others where significant oscillations appear, which can lead to failures in the system. From these results we propose an alternative for the BitTorrent protocol, which leads to a more stable system. Through simulations and mathematical modeling, we evaluate the performance improvements provided by this variant.

Keywords: Peer-to-peer networks. BitTorrent. Simulations. Modelization. Choking algorithms.
KOZYNSKI, Fabián; FERRAGUT, Andrés; PAGANINI, Fernando. Reducción de osciláciones én BitTorrent mediante mecanismos de unchoking preferencial. Informática na Educação: teoria \& prática, Porto Alegre, v. 14, n. 1, p. 29-41, jan./jun. 2011 .

\author{
Fabián Kozynski \\ Universidad ORT Uruguay \\ Andrés Ferragut \\ Universidad ORT Uruguay \\ Fernando Paganini \\ Universidad ORT Uruguay
}

\section{Introducción}

$\mathrm{B}$

itTorrent (COHEN, 2003; COHEN, 2008; Theory.org, 2010) es un sistema peer-to-peer de distribución de contenido en el cual un conjunto de usuarios busca descargar un conjunto determinado de archivos. Utilizar un sistema peer-to-peer permite que la capacidad de servicio del sistema escale junto con la demanda del mismo. Para lograr esto, los usuarios intercambian partes del contenido entre sí hasta lograr completar la descarga. Aquellos peers que se encuentran en la primer instancia, es decir, todavía están descargando contenido del sistema, son llamados leechers. Los peers que ya completaron la descarga son llamados seeders. Estos últimos tienen la posibilidad de permanecer en el sistema tanto tiempo como deseen, facilitando así el proceso de otros usuarios.

El protocolo BitTorrent consiste en una serie de mecanismos y heurísticas. Todo el sistema de archivos se divide en piezas (chunks), las cuales son indexadas para poder identificarlas y cuentan con un control 
de integridad. Cada usuario busca conseguir todas las piezas solicitándolas a aquellos peers con los que se encuentra conectado. Cuando un leecher descarga una de estas piezas en forma completa, avisa a todos sus contactos que la posee y que puede ser solicitada. Esto, junto con el envío de un bitfield marcando las piezas que tienen al momento del contacto inicial, permite a un peer mantener la información de estado, la lista de piezas que posee cada uno de sus contactos.

A partir de las solicitudes recibidas, cada peer decide a cuales otros peers autoriza a descargar durante el siguiente intervalo de tiempo (generalmente $10 s$ ), proceso llamado unchoking. En esta selección se incorpora un mecanismo conocido como titfor-tat (TFT), para incentivar la compartición y evitar los free-riders que no contribuyen al sistema. Dicho mecanismo consiste en autorizar a descargar (unchoke) a aquellos leechers que durante una ventana de tiempo han contribuido más contenido al peer en cuestión. Se agregan además autorizaciones extra (optimistic unchoking) de un peer al azar, para detectar potenciales nuevos colaboradores.

Una pregunta natural es si estos mecanismos de base heurística logran un desempeño adecuado, especialmente en una situación dinámica en la que peers entran y salen del sistema. Para ello se ha recurrido al modelado matemático, comenzando por De Veciana y Yang (2003) con modelos Markovianos estudiados en forma numérica. Qiu y Srikant (2004) presentn un modelo de fluido inspirado en el de De Veciana y Yang (2003), y se obtienen resultados analíticos en cuanto al equilibrio al que llega el sistema BitTorrent, y su estabilidad. Este modelo se resume en la Sección 2 .

Un primer objetivo del presente trabajo es la evaluación por simulaciones de BitTorrent, buscando validar su desempeño y las conclusiones de este modelo. En la Sección 3 se describen estas simulaciones, basadas en la biblioteca (EGER et al., 2007) para NS-2. Observamos que según los parámetros empleados, el sistema puede comportarse como es debido (operando alrededor del equilibrio previsto por Qiu y Srikant (2004)), o por el contrario generar graves oscilaciones que llevan a la falla, en particular la llamada muerte del torrent. Concluímos por tanto que el modelo tiene limitaciones en su poder predictivo, y lo que es más grave: el BitTorrent práctico también tiene problemas.

Un estudio detallado de la situación de falla nos lleva a proponer una variante al protocolo BitTorrent, descrita en la Sección 4. Esta variante es estudiada por simulación, y también en la Sección 5 a través del modelado matemático. Observamos que efectivamente se logra una mejora de desempeño en las situaciones problemáticas. Las conclusiones y líneas de trabajo a futuro se describen en la Sección 6.

\section{Modelo fluido de Qiu y Srikant}

Para modelar un sistema de BitTorrent, Qiu y Srikant proponen un modelo fluido. A partir de dicho modelo, obtienen una serie de resultados, los cuales se utilizarán como base para las siguientes secciones. A continuación se incluye una breve descripción del modelo, así como los resultados principales respecto de los equilibrios del mismo.

El modelo se encuentra basado en dos colas en cascada. Una de las colas, representada por $x$, corresponde a la cantidad de leechers en el sistema. La otra, $y$, corresponde a la cantidad de seeders. Ambas pueden tomar valores en el intervalo $[0,+\infty)$. La dinámica de estas dos variables se modela como se describe a continuación:

$$
\begin{aligned}
& \dot{x}=\lambda-\min \{c x, \mu(y+\eta x)\} \\
& \dot{y}=\min \{c x, \mu(y+\eta x)\}-\gamma y
\end{aligned}
$$

- $\lambda$ es la tasa de arribo de leechers, que llegan sin ningún contenido.

- $\mu$ es la capacidad de subida de un peer en archivos por segundo, por tanto $\mu(y+\eta x)$ es la capacidad de subida total del sistema, donde se incluye un parámetro $\eta \in[0,1]$ que representa la eficiencia de la compartición entre leechers.

- $c>\mu$ es la capacidad máxima de bajada de un peer. La velocidad de bajada total está entonces determinada por 
$\min \{\mathrm{cx}, \mu(\mathrm{y}+\eta \mathrm{x})\}$; ésta determina la tasa de pasaje de leechers a seeders, como se indica en (1).

- También se incluye una tasa $\theta$ de abandono de los leechers, y una tasa $\gamma$ de abandono de los seeders.

Se analizarán los equilibrios en el caso en que los leechers no abandonan el sistema antes de completar la descarga $(\theta=0)$. El punto de equilibrio de este sistema tiene dos casos, dependiendo de los valores de los parámetros:

(i) Si $\frac{1}{\mu}<\frac{1}{\gamma}+\frac{\eta}{c}$, el equilibrio es $x^{*}=\frac{\lambda}{c} \quad y^{*}=\frac{\lambda}{\gamma}$

y está limitado por la capacidad de bajada $c$. Es decir, el mínimo de (1) ocurre en $c x^{*}$.

(ii) Si $\frac{1}{\mu}>\frac{1}{\gamma}+\frac{\eta}{c}$, el equilibrio es $x^{*}=\frac{\lambda}{\eta}\left(\frac{1}{\mu}-\frac{1}{\gamma}\right) \quad y^{*}=\frac{\lambda}{\gamma}$

y está limitado por la capacidad de subida.

Notar que para $c » \mu$, común en la práctica, los casos anteriores son aproximadamente (i) $\gamma<\mu$ y (ii) $\gamma>\mu$. El caso (i) se da cuando el tiempo medio $\frac{1}{\gamma}$ de permanencia de un seeder supera al tiempo $\frac{1}{\mu}$ necesario para reponer un archivo al sistema, generando otro seeder. Por tanto, aquí los seeders solos podrían sostener la carga, y se acumulan en el sistema hasta saturar la capacidad de bajada. El caso (ii) es el contrario: aquí los seeders solos no son suficientes para atender la demanda, y la compartición entre leechers se vuelve esencial. La cantidad de leechers en el sistema se ajusta en el equilibrio para proveer la tasa de subida faltante.

En Qiu y Srikant (2004) se prueba que en ambos casos el equilibrio es localmente estable. Más aún, Qiu y Sang (2008) prueban la estabilidad global del sistema nolineal conmutado (1).

También puede extraerse del modelo el tiempo medio de descarga del archivo. En el caso (i) es simplemente $\bar{T}=\frac{1}{c}$, en el caso (ii) se obtiene

$$
\bar{T}=\frac{1}{\eta}\left(\frac{1}{\mu}-\frac{1}{\gamma}\right) .
$$

\section{Análisis por simulaciones}

Para validar el modelo mencionado en la sección anterior se realizaron simulaciones en un sistema que reproduce los algoritmos del protocolo BitTorrent. Para esto se utilizó una biblioteca para BitTorrent implementada en NS-2 (EGER et al., 2007).

Las simulaciones se realizaron tomando valores para los parámetros que podrían ser los que se encuentren en un sistema real compuesto por usuarios domésticos. A partir de dichas simulaciones se relevó la evolución de las cantidades de seeders y leechers a lo largo de la simulación, y la evolución de la subida y la descarga para cada uno de los peers.

Todas las simulaciones se llevaron a cabo en un sistema compuesto por peers ubicados en una topología de estrella, todos conectados a un nodo central. Dichos peers intentan descargar un archivo de $100 \mathrm{MB}$ dividido en 400 piezas. Todos los leechers ingresan al sistema sin contenido y solo se van una vez que completaron la descarga, luego de permanecer un tiempo como seeders.

Para simular una situación real se utilizan arribos y partidas aleatorios. Para los arribos de leechers se utilizó un proceso de Poisson de parámetro $\lambda$, y para las partidas de seeders se tomó un tiempo exponencial de parámetro $\gamma$. El modelo determinístico (1) permite estudiar el comportamiento medio de este sistema aleatorio. Las oscilaciones alrededor de este valor medio son estudiadas por Qiu y Srikant (2004) considerando un modelo de ruido Browniano cosa que no haremos aquí por simplicidad. Por lo tanto nuestra validación se basará en los valores medios de las variables simuladas.

\subsection{Caso limitado por capacidad de bajada}

Primero consideramos el caso (i) de la Sección 2 (aproximadamente, $\gamma<\mu$ ), donde el modelo predice un equilibrio saturado por la capacidad $c$ de bajada. Esta predicción se 
verificó efectivamente en numerosas simulaciones. A continuación mostramos un caso, con los siguientes parámetros: $\lambda=0.01^{\text {arribos }} / \mathrm{s}, \quad \gamma=10^{-4} \mathrm{~s}^{-1} ; \quad \mu \approx 1.5 \cdot 10^{-4} \mathrm{~s}^{-1}$, correspondiente a una velocidad de subida de $\quad 128 \mathrm{Kbps} ; \quad c=8 \mu \approx 1.2 \cdot 10^{-3} \mathrm{~s}^{-1}$, correspondiente a una velocidad de bajada de $1 \mathrm{Mbps}$. La simulación se comenzó con una cantidad de 100 seeders y 10 leechers con contenido aleatorio.

En la Figura 1 se observa la evolución del sistema. Comparando los valores medios tanto de seeders como de leechers en la simulación, se observa que éstos son similares a los del modelo. La cantidad de leechers en el equilibrio es de 8.9 , cuando $x^{*}=\frac{\lambda}{c}=8.19$; y la cantidad de seeders en el equilibrio es de 100.58 , siendo $y^{*}=\frac{\lambda}{\gamma}=100$.

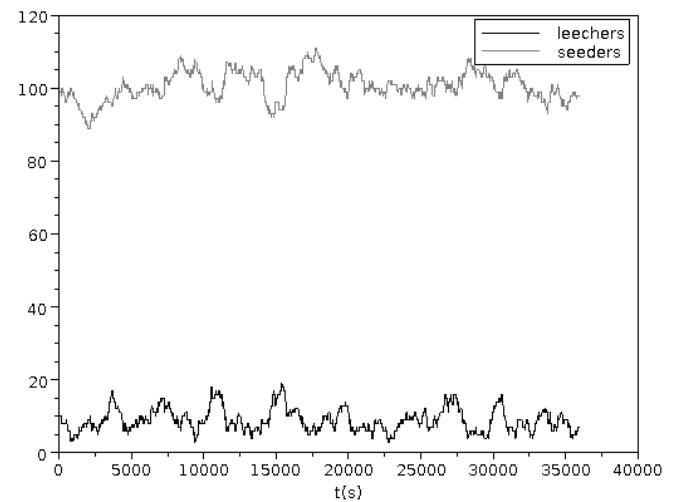

Figura 1 - Evolución de un sistema en torno a un equilibrio limitado por capacidad de bajada.

También se midió empíricamente la velocidad de subida y de bajada de los distintos peers, a través de la gráfica de la Figura 2. Allí se muestra el volumen de descarga en función del tiempo para cada peer, a partir del momento de ingreso al sistema (tiempo propio). Por lo tanto las pendientes de las curvas corresponden a las velocidades de subida y bajada. Se verifica que la velocidad de bajada es uniforme entre los peers, y próxima al límite de 1 Mbps. Por otra parte, la velocidad de subida presenta mayor dispersión, y está por debajo del límite de $128 \mathrm{Kbps}$; esto es consistente con el modelo, estamos en el caso en que hay sobrante en la capacidad de subida.

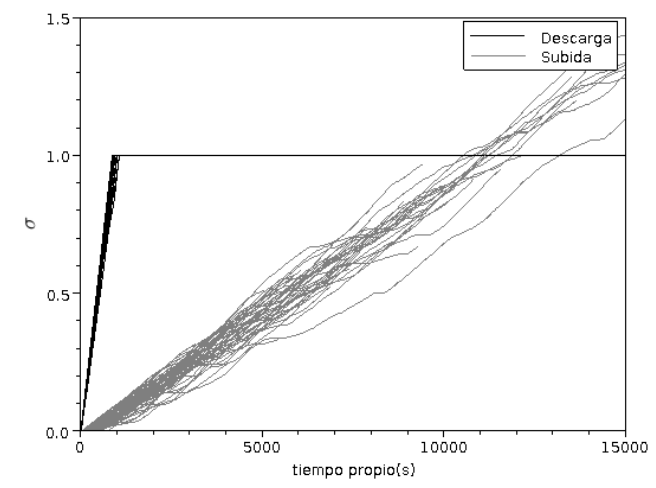

Figura 2 - Transferencia acumulada de distintos peers, caso limitado por capacidad de bajada.

\subsection{Caso limitado por capacidad de subida}

Se estudia ahora el caso (ii) de la
Sección 2; se tomó $\mu \approx 3 \cdot 10^{-4} \mathrm{arch} / \mathrm{s}$ (correspondiente a una velocidad de subida de $256 \mathrm{Kbps}$ ) y $\gamma=5 \cdot 10^{-4} \mathrm{~s}^{-1}$, lo cual corresponde a un tiempo medio de estadía de los seeders de aproximadamente media hora. Se cumple entonces $\mu<\gamma$, y también la restricción más estricta (ii) para la capacidad $c=8 \mu$ utilizada.

En esta simulación $\lambda=0.01^{\text {arribos }} / \mathrm{s} ;$ se comienza con 40 seeders y ningún leecher. En la Figura 3 se observa la evolución de los seeders y leechers a lo largo de la simulación. Ambos valores evolucionan presentando oscilaciones en torno a un equilibrio. Si bien el ruido aquí es mayor, el valor promedio de los seeders observados es 21, aproximadamente consistente con la cantidad $\frac{\lambda}{\gamma}=20$ indicada por el modelo.

Para comparar la cantidad media de leechers con el modelo, es necesario conocer el parámetro $\eta$ de eficiencia de la compartición. Estudiamos para ello las tasas de transferencia de los distintos peers a lo largo de la simulación. La Figura 4 muestra otra vez transferencias acumuladas. 


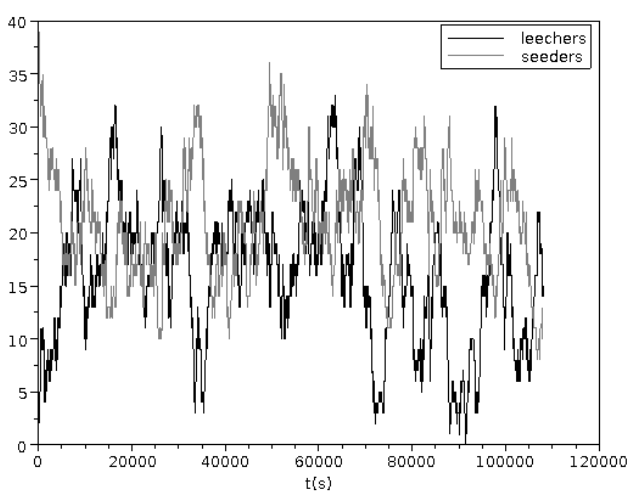

Figura 3 - Evolución de un sistema en torno a un equilibrio limitado por capacidad de subida.

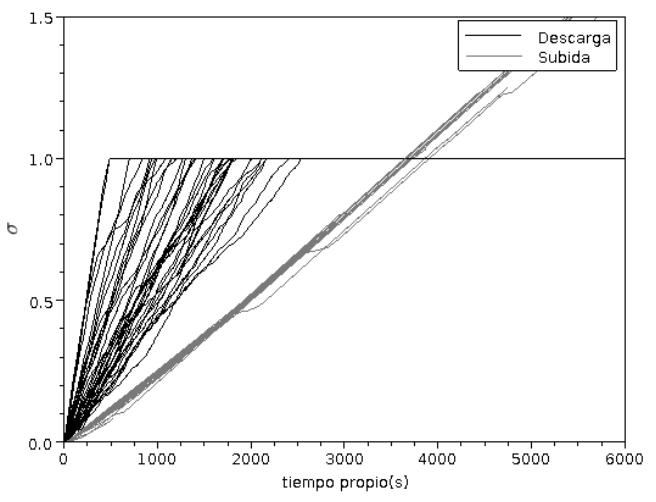

Figura 4 - Transferencia acumulada de distintos peers, caso limitado por capacidad de subida.

Se observa ahora que las tasas de subida son uniformes entre peers, consistente con la saturación por subida. El valor obtenido empíricamente es $\mu^{\prime} \approx 0.9 \mu$; podría pensarse en atribuir esto al parámetro $\eta$ de eficiencia; sin embargo, una observación cuidadosa muestra que la velocidad de subida permanece constante, incluso después de que el peer se transformó en seeder. Por lo tanto, el factor de eficiencia debe tomarse como $\eta \approx 1$ (consistente con un análisis de Qiu y Srikant (2004)), y la pérdida del $10 \%$ se atribuye en cambio a un overhead general del sistema.

En ese sentido, notar que las gráficas de la Figura 4 se realizaron a partir de trazas de transferencias de carga útil (es decir, mensajes PIECE de BitTorrent), y no se encuentran considerados tanto los mensajes de control del protocolo como los distintos encabezados de las capas inferiores.

Si contemplamos todo esto reemplazando $\mu$ por $\mu^{\prime} \approx 0.9 \mu$ en (2), y tomamos $\eta=1$, el modelo predice una cantidad de leechers en el equilibrio de $\lambda\left(\frac{1}{\mu}-\frac{1}{\gamma}\right) \approx 16.4$, mientras que la cantidad media de leechers en la simulación es aproximadamente 16 . Por lo tanto concluimos que una vez más el modelo está validado en este caso.

\subsection{Limitaciones del modelo}

Consideramos ahora otra simulación, con parámetros $\quad \lambda=0.01^{\text {arribos }} / \mathrm{s}, \quad \gamma=10^{-3} \mathrm{~s}^{-1}$ (reduciendo el tiempo medio de permanencia de los seeders a $1000 s$ ) y reduciendo $\quad \mu \approx 7 \cdot 10^{-5} \mathrm{arch} / \mathrm{s} \quad$ (64 Kbps). Estamos por tanto en un caso de $\gamma » \mu$, en el que el modelo predice un equilibrio saturado por subida, y sostenido principalmente por la compartición de los leechers. En cambio, en la simulación se observa algo muy distinto: el torrent "muere" al poco tiempo, hecho que se ve en la Figura 5. Esto significa que en cierto momento el sistema se queda sin seeders, y los leechers restantes no contienen entre todos una copia completa del archivo. Esta patología no está prevista en el modelo, ya que éste no hace referencia a las piezas.

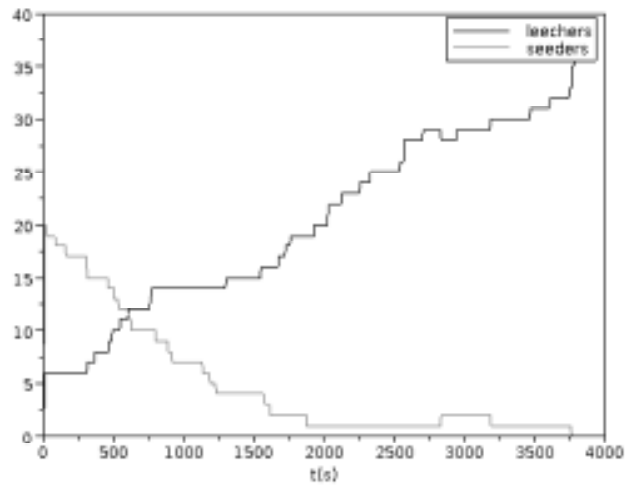

Figura 5 - Evolución de un torrent que "muere".

En esta simulación, la cual se comenzó con 20 seeders y 5 leechers (con cantidad y distribución aleatoria de piezas), se llegó a 
un punto en el que no habían más seeders en el sistema. En este momento, 16 de las piezas no se encontraban presentes en ningún leecher.

Para poder proseguir el estudio más allá del punto de muerte, se agregó un seeder permanente (servidor), el cual mantiene todas las piezas disponibles y evita la muerte del torrent. Se espera que el agregado de este servidor no tenga mucho impacto en el equilibrio, donde en este caso se contaría con $\frac{\lambda}{\gamma}=10$ seeders.

Sin embargo, al realizar una nueva simulación contando con el servidor no se logra este equilibrio. Como muestra la Figura 6 tenemos en su lugar una oscilación cuasi-periódica, con picos de creación y partida de seeders, y largos intervalos en los que el sistema queda vacío de seeders, dependiendo exclusivamente del servidor. Estas oscilaciones son demasiado sistemáticas para atribuirlas exclusivamente al ruido, indican una limitación del modelo.

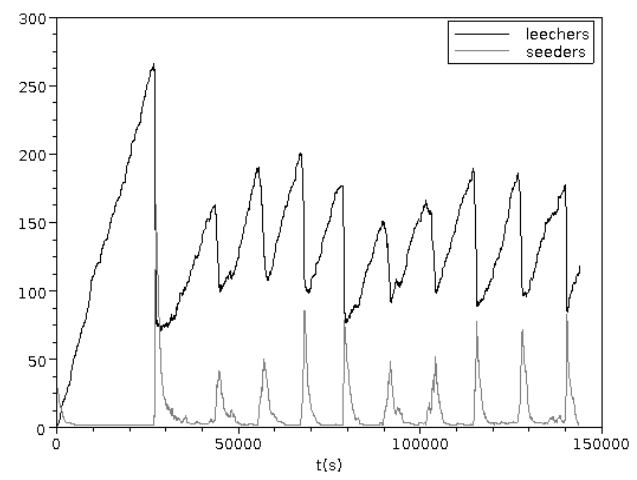

Figura 6 - Evolución de un sistema con servidor que presenta oscilaciones.

Los picos de generación de seeders corresponden a un conjunto de leechers que culminan la descarga en un corto intervalo de tiempo. Dado que las llegadas de los leechers están distribuidas en el tiempo, esto implica que hay leechers que llegan más tarde y logran equipararse a los que llegaron antes, a través de una velocidad de descarga mayor. Esto en consistente con las gráficas de descarga de la Figura 7, correspondientes a un ciclo determinado, donde se ven velocidades de bajada no uniformes entre peers y con variaciones a lo largo del tiempo.

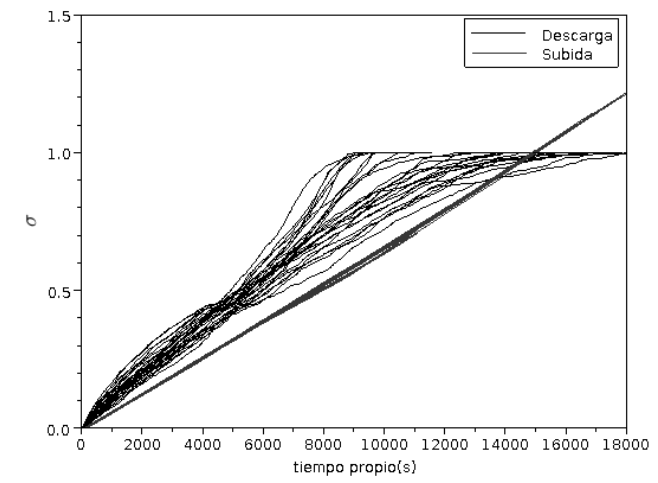

Figura 7 - Transferencia acumulada de distintos peers, en un sistema con ciclo límite.

Notar que la velocidad de subida de todos los peers es prácticamente la misma y constante durante toda la evolución; se mantiene la eficiencia observada en la simulación anterior.

La discrepancia observada entre modelo y simulación se atribuye entonces a dos aspectos no considerados en el modelo:

- La distribución desigual del ancho de banda entre peers con diferente grado de descarga;

- La entidad específica de las piezas intercambiadas, como se discute más abajo.

\subsection{Estudio detallado de la falla observada en BitTorrent}

Más allá de las limitaciones del modelo en su capacidad de predecir el comportamiento real, este comportamiento en sí mismo resulta problemático, ya que se observa la muerte del torrent. Esto ocurre en una situación en la que debería poder sostenerse el sistema a través de la compartición de leechers. Y si se evita la muerte a través de los servidores, estos juegan un papel demasiado central, sosteniendo el sistema durante un largo período, llevando a un comportamiento oscilatorio no deseable. Tenemos por tanto una limitación inherente al protocolo BitTorrent.

Observamos detenidamente la simulación con servidores, con especial atención a la descarga de aquellos leechers que se encuentran más tiempo en el sistema, a 
través de la evolución de los respectivos bitfield. Se detecta que existen usuarios con una gran cantidad de piezas (faltándoles no más de 5), los cuales durante mucho tiempo no logran completar ninguna pieza. Esto se debe a que dichas piezas no se encuentran en el sistema y sólo las poseen los servidores. Por lo tanto, necesitan que éstos les realicen un unchoke para obtenerlas y que así ingresar al sistema.

Se hace notar que BitTorrent establece la preferencia a solicitar piezas más escasas, justamente para atender este tipo de problema. Sin embargo, para conseguir las piezas faltantes, se pueden presentar dos situaciones. Por un lado, puede que los leechers interesados no consigan unchoke de los servidores, que son los únicos que poseen las piezas que necesitan. Por otro lado, los leechers a los que sí realizan unchoke los servidores, también ven otras piezas como escasas entonces no necesariamente solicitan las piezas faltantes. Estas dos situaciones llevan a que las piezas faltantes demoren más tiempo en ingresar al sistema.

Detectamos entonces una debilidad del sistema BitTorrent cuando opera con una proporción baja de seeders, es decir cuando los usuarios son egoístas y se retiran poco después de terminar su descarga. Si bien en teoría este sistema podría funcionar sosteniéndose principalmente en la compartición entre leechers, en la práctica se observa una tendencia a sincronizar las partidas de los seeders que conduce a la muerte del torrent.

\section{BitTorrent Elitista}

El problema descrito en la sección anterior surge a partir de leechers que se estancan en la descarga de las últimas piezas debido a que no las consiguen. A partir de esto se decidió modificar el algoritmo de unchoke presente en el protocolo de forma de solucionar este problema.

La propuesta, que llamamos BitTorrent elitista, es que los peers dediquen sus puestos de subida (con la excepción del Optimistic unchoke) a aquellos peers que observan con la mayor cantidad de piezas.
De esta manera, el sistema siempre buscaría crear seeders a partir de aquellos leechers que se encuentran cercanos a terminar la descarga. Con esta modificación se espera que se generen seeders a una tasa más estable, evitándose las avalanchas observadas anteriormente.

La modificación fue implementada en NS2 modificando la aplicación (cliente) de Eger et al. (2007), y se realizaron simulaciones cubriendo los distintos casos de funcionamiento.

\subsection{Resultados de simulación}

En términos generales, puede decirse que en los casos en que BitTorrent llegaba a un equilibrio, la variante elitista también lo hace. Además, las cantidades de leechers y seeders en equilibrio no varían significativamente respecto de los valores anteriores. En la Sección 5 justificaremos este hecho mediante un modelo.

En estos casos en que BitTorrent se comportaba razonablemente bien en valores medios, igual la alternativa elitista mejora el desempeño en cuanto al comportamiento dinámico, reduciendo la magnitud de las oscilaciones alrededor del equilibrio. Esto se observa en la serie de simulaciones de la Figura 8.

Allí se representa la evolución de diversas simulaciones realizadas con los mismos valores de $\lambda\left(0.01^{\text {arribos }} / s\right)$ y $\mu$ (equivalente a $256 \mathrm{Kbps}$ ), variando el valor de $\gamma$. La Figura 8a muestra las simulaciones realizadas con el cliente estándar, mientras que la Figura 8b muestra las simulaciones realizadas con el cliente elitista. En ambos casos los valores de $\gamma$ van desde $10^{-3} s^{-1}$ hasta $3 \cdot 10^{-3} s^{-1}$; la evolución de los leechers se representa en oscuro mientras que la de los seeders se representa en.

Se puede ver que al utilizar el cliente elitista las oscilaciones se vuelven menos definidas. A su vez, los sistemas no pasan la mayor parte del tiempo trabajando solamente con los servidores, sino que en todo momento se tiene por lo menos un seeder común. 
Comentamos por otra parte que en todos los casos simulados el cliente elitista mantiene una compartición eficiente, donde la velocidad de subida de todos los peers se encuentra saturada.
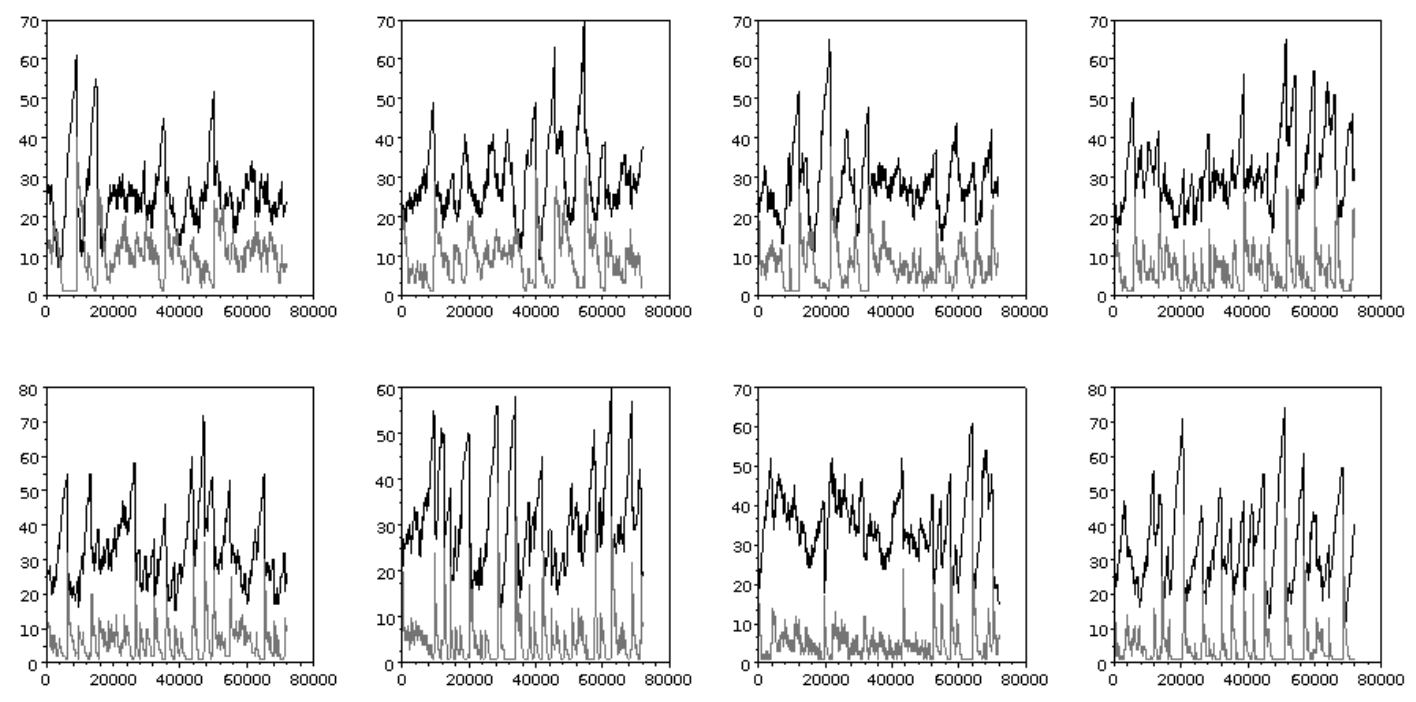

Figura 8a - Simulaciones con cliente estándar.
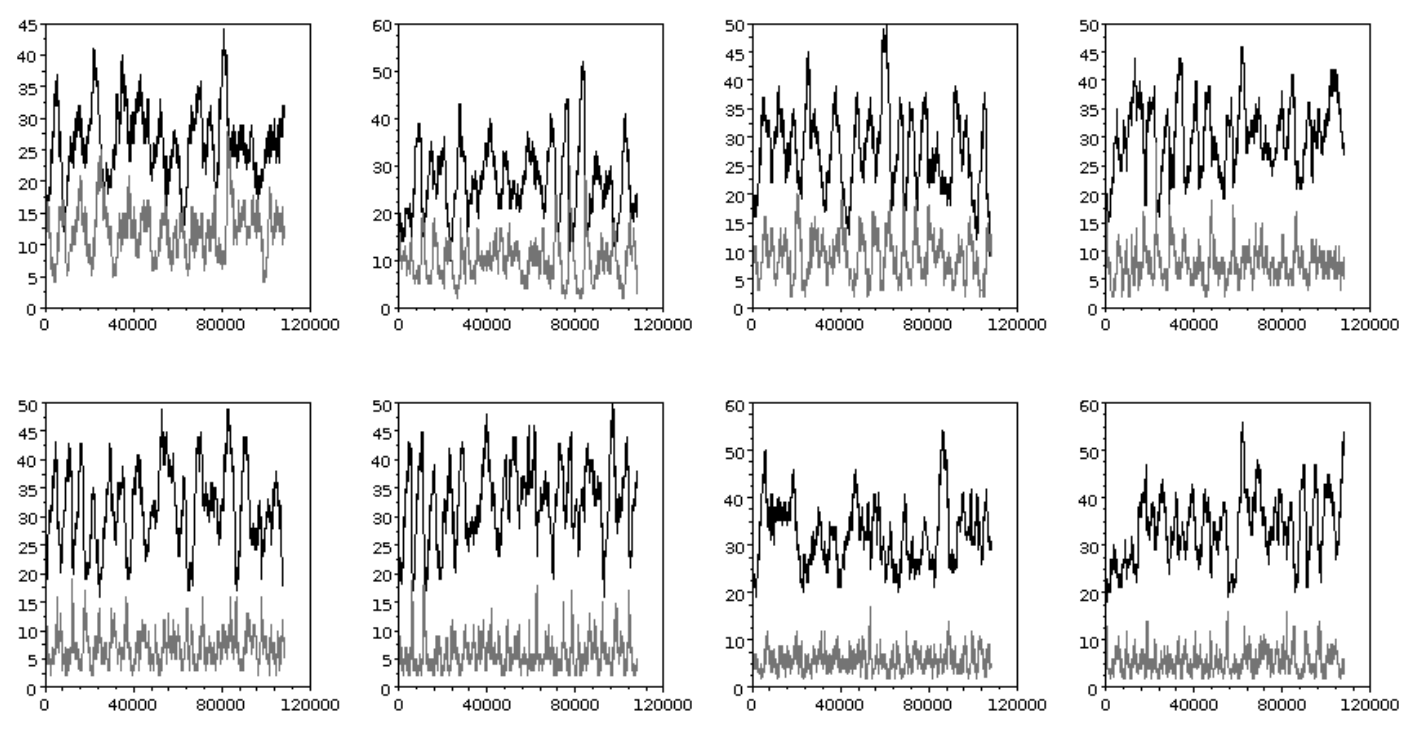

Figura $8 b$ - Simulaciones con cliente elitista.

Figuras 8 - Comparación de clientes.

Una vez verificado que el cliente modificado no empeora las cosas, pasamos a ver si efectivamente logra una mejora en los casos problemáticos. En la Figura 9 se muestra la evolución con los parámetros de la Sección 3.3. Si bien sigue habiendo oscilaciones, hay una gran diferencia: en este caso, existen leechers culminando la descarga en todo momento, lo que lleva a un comportamiento más suave del sistema con un número de seeders que no llega casi nunca a cero (exceptuando el transitorio 
inicial); esto implica que la supervivencia del torrent no depende crucialmente de los servidores.

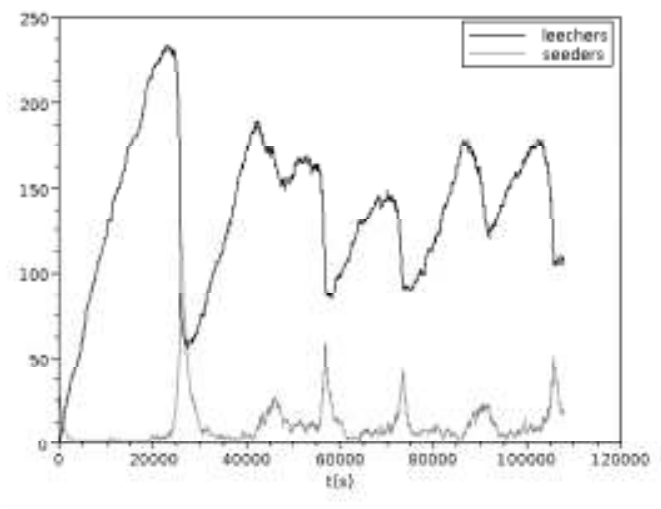

Figura 9 - Evolución de un sistema patológico utilizando un cliente elitista.

\subsection{Mejora en la justicia del sistema}

Superficialmente, podría pensarse que un sistema elitista que favorece los unchoke a leechers más "ricos" puede generar injusticias, por ejemplo en los tiempos de descarga. Sin embargo, ocurre lo contrario.

En la Figura 10 se observa la distribución de las duraciones de las descargas en dos simulaciones con los mismos parámetros, utilizando en cada una un cliente distinto. En ambas simulaciones el sistema trabaja en torno a un equilibrio, con parámetros similares a los de la simulación de la Figura 3.
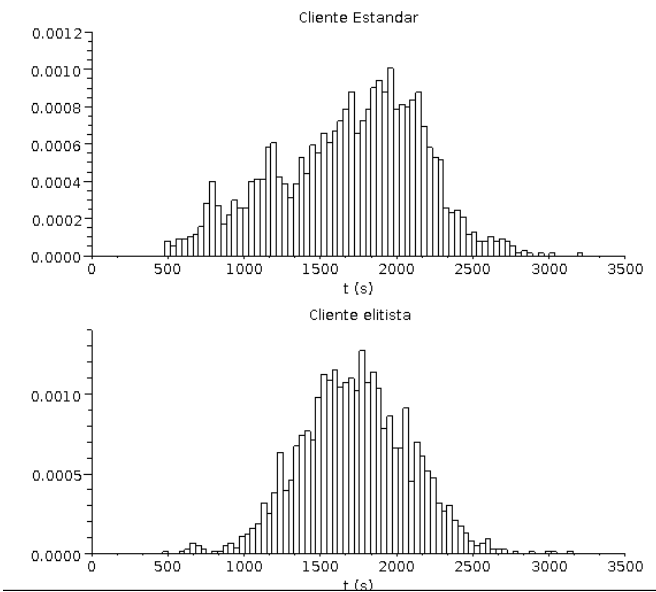

Figura 10 - Duración de las descargas utilizando clientes estándar y clientes elitistas.
Una primera observación es que los valores medios de los tiempos de descarga no cambian, son consistentes con la fórmula (3) (reemplazando con $\mu^{\prime} \approx 0.9 \mu$ ). Esto es también consistente con el número medio de peers en equilibrio, que como se dijo tampoco cambia.

La diferencia principal observada en los histogramas es en la varianza de los tiempos de descarga, que disminuye claramente en el cliente elitista; se pasa de una desviación estándar de $475 \mathrm{~s}$ para clientes comunes a un valor de $355 \mathrm{~s}$ para los elitistas. Esta disminución de la varianza mejora la justicia del sistema: los tiempos de descarga son más parejos entre los distintos leechers.

La explicación de esta menor varianza se atribuye a la disminución de las oscilaciones. En un sistema saturado por subida, la velocidad de descarga que experimentan los leechers en un cierto período de tiempo es proporcional a la cantidad de peers en el sistema durante dicho período. Si esta cantidad no varía significativamente en el tiempo, leechers que ingresan en distintos momentos de la simulación experimentan una red de capacidad más estable. Si bien esta capacidad ahora no se distribuye uniformemente entre los leechers, sino que hay un sesgo hacia los "ricos", esto no afecta el tiempo total de descarga, ya que todo peer aprovecha esta ventaja sobre el final de su descarga.

En otras palabras, ahora los leechers tienen una fase en que no son privilegiados y avanzan lentamente, y a partir de cierto momento son privilegiados y terminan rápidamente. Estas dos fases pueden observarse en la gráfica de descarga de la Figura 11. En esa figura, en la etapa privilegiada los leechers saturan por capacidad de bajada, logrando un desempeño confiable; las variaciones entre leechers se atribuyen principalmente a la primer fase, por lo que no sorprende que la varianza total sea de menor entidad.

En la Tabla 1 se observan las diferencias entre las varianzas de los tiempos de descarga entre ambos clientes para las simulaciones de la Figura 8. Se puede observar que al cambiar de cliente, el 
tiempo medio de descarga no varía significativamente (en relación con la diferencia observada en la varianza).

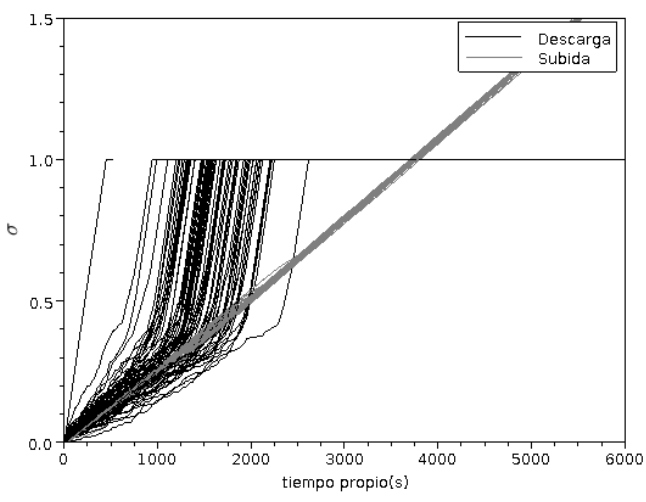

Figura 11 - Evolución de la descarga de distintos peers en un sistema elitista.

Tabla 1 - Resumen de la disminución de la varianza

\begin{tabular}{cccccc}
\hline$\lambda$ & $\gamma$ & \multicolumn{2}{c}{ Estándar } & \multicolumn{2}{c}{ Elitista } \\
\cline { 3 - 6 }$($ arribos $/ s)$ & $\left(10^{-3} s^{-1}\right)$ & $\bar{T}(s)$ & $\sigma(s)$ & $\bar{T}(s)$ & $\sigma(s)$ \\
\hline \multirow{6}{*}{0.01} & 1.0 & 2603 & 632 & 2538 & 418 \\
& 1.2 & 2823 & 568 & 2722 & 491 \\
& 1.5 & 2869 & 555 & 2824 & 448 \\
& 1.8 & 3130 & 579 & 2885 & 461 \\
& 2.0 & 3117 & 668 & 3070 & 392 \\
& 2.5 & 3230 & 775 & 3195 & 399 \\
& 2.8 & 3282 & 562 & 3196 & 355 \\
& 3.0 & 3302 & 984 & 3219 & 349 \\
\hline
\end{tabular}

\subsection{Alternativa "Altruista"}

Para enfatizar aún más que las evaluaciones de justicia no siempre coinciden con lo intuitivo, estudiamos en esta sección la estrategia opuesta a la elitista. Es decir, todos los peers dedican todos sus unchokes (sin contar el Optimistic) a aquellos leechers que poseen menor cantidad de contenido descargado.

Este método toma elementos de "justicia max-min" o "Rawlsiana" muy invocados en las redes: en muchas situaciones, favorecer al que tiene menos tiende a una distribución igualitaria de los recursos.

En ésta situación dinámica, la alternativa no resulta recomendable, como demostramos a continuación a partir de las simulaciones de las Figuras 12 y 13, para distintos valores de los parámetros.
La Figura 12 utiliza los mismos valores de $\gamma$ y $\mu$ que la simulación correspondiente a la Figura 3, con un valor de $\lambda=0.02 \operatorname{arribos} / \mathrm{s}$. Se observa que en este caso las oscilaciones han aumentado dramáticamente, lo cual inevitablemente conduce a mayores diferencias (injusticia) en los tiempos de descargas de los peers de acuerdo al momento de su entrada en el sistema.

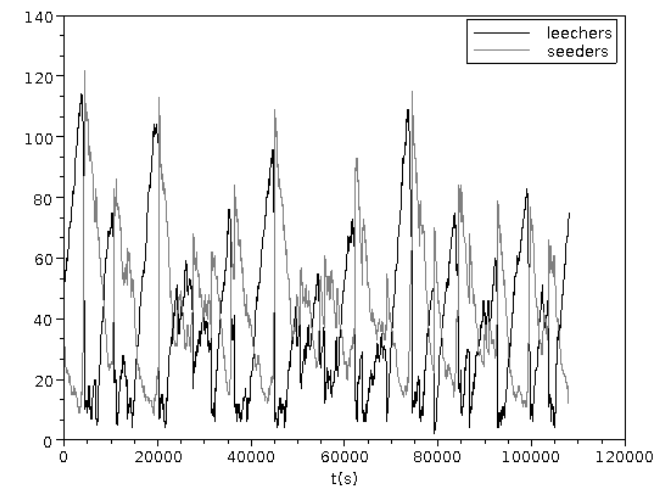

Figura 12 - Evolución del sistema "altruista", caso torrent estable.

La Figura 13 corresponde a los parámetros de la Figura 6, situación en el cual el BitTorrent standard ya tenía un comportamiento oscilatorio. Vemos que se acentúan en este caso las oscilaciones, en particular el sistema pasa casi todo el tiempo dependiendo del servidor fijo que se agregó para evitar la muerte del Torrent.

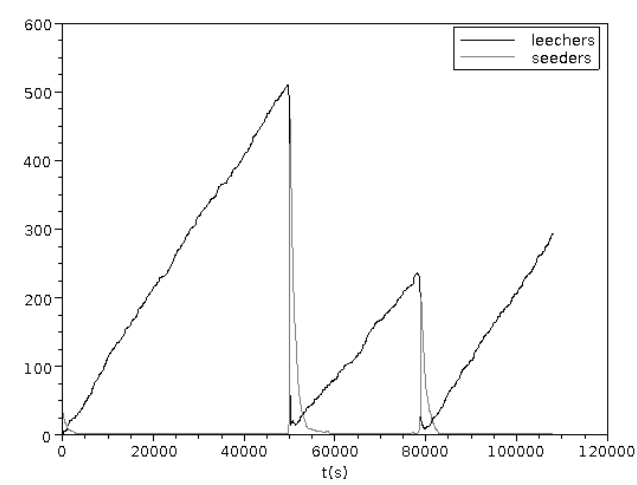

Figura 13 - Evolución del sistema "altruista", caso oscilatorio.

La explicación de esta patología es directa: dar prioridad a los peers con pocas piezas en un ambiente donde permanentemente llegan leechers nuevos, 
inevitablemente conduce a la acumulación de leechers, postergando la culminación de todos. En cierto momento la capacidad excesiva acumulada posibilita la terminación de un gran número de peers en un corto intervalo de tiempo, después del cual el sistema se vacía. Lejos de lograrse justicia, se han acentuado por este método las patologías existentes, por lo cual esta alternativa no será considerada más.

\section{Modelado del sistema Elitista}

Para entender mejor el cliente elitista, se realizará un modelo que cuente con algo de información de la distribución de los leechers en el sistema según la cantidad de contenido descargado. Nos concentramos en el caso (ii) de la Sección 2, restringido por la capacidad de subida, con $\mu<\gamma$, y con capacidad de bajada $c$ suficientemente grande como para ser no ser incluida en el modelo.

Para esto, se dividirá el estado $x$ de los leechers del modelo introducido en la Sección 2 en dos estados $n_{1}$ y $n_{2}$. Dichos estados corresponderán a leechers que tienen menos de la mitad del contenido descargado $\left(n_{1}\right)$ y más de la mitad del contenido descargado $\left(n_{2}\right)$.

Suponiendo que los leechers comparten contenido con máxima eficiencia, la velocidad de descarga total será $\mu\left(y+n_{1}+n_{2}\right)$ archivos por segundo o equivalentemente, $2 \mu\left(y+n_{1}+n_{2}\right)$ mitades de archivo por segundo. Esta última expresión es la relevante, ya que los avances de $n_{1}$ a $n_{2}$, y de $n_{2}$ a $y$, corresponden a descargar medio archivo.

Para el cliente elitista, dicha capacidad total no se distribuirá en forma equitativa entre ambos estados. Se introduce la variable $\rho$, la cual representa el cociente entre el porcentaje de capacidad correspondiente a $n_{2}$ y el correspondiente a $n_{1}$. A partir de esto, el sistema de ecuaciones diferenciales que determina la dinámica del sistema queda así:

$$
\left\{\begin{array}{l}
\dot{n}_{1}=\lambda-2 \mu\left(y+n_{1}+n_{2}\right) \frac{n_{1}}{n_{1}+\rho n_{2}} \\
\dot{n}_{2}=2 \mu\left(y+n_{1}+n_{2}\right)\left[\frac{n_{1}}{n_{1}+\rho n_{2}}-\frac{\rho n_{2}}{n_{1}+\rho n_{2}}\right] \\
\dot{y}=2 \mu\left(y+n_{1}+n_{2}\right) \frac{\rho n_{2}}{n_{1}+\rho n_{2}}-\gamma y
\end{array}\right.
$$

A partir de dicho sistema, es posible obtener el estado de equilibrio:

$$
\begin{aligned}
n_{1}^{*} & =\frac{\lambda \rho}{\rho+1}\left(\frac{1}{\mu}-\frac{1}{\gamma}\right) \\
n_{2}^{*} & =\frac{\lambda}{\rho+1}\left(\frac{1}{\mu}-\frac{1}{\gamma}\right) \\
y^{*} & =\frac{\lambda}{\gamma}
\end{aligned}
$$

Se puede observar que la cantidad total de leechers, $n_{1}^{*}+n_{2}^{*}$ es independiente de $\rho$ y es igual al valor de $x^{*}$ dado en (2) para un sistema de eficiencia $\eta=1$. Por lo tanto, la forma en que se destine el ancho de banda disponible no modifica la cantidad de leechers en el equilibrio. Además, la cantidad de seeders en el equilibrio sigue siendo $\frac{\lambda}{\gamma}$. Estos resultados pueden generalizarse para modelos con más estados intermedios y comparticiones más generales (ver KOZYNSKI, 2011).

Para estudiar la dinámica de este sistema, se realiza la linealización en torno al punto de equilibrio. Normalizando el sistema con $\mu=1$, se obtiene la siguiente matriz jacobiana

$$
J=\left[\begin{array}{ccc}
\frac{(2-3 \gamma) \rho-\gamma}{2(\gamma-1) \rho} & \frac{\gamma(\rho-1)+2}{2(\gamma-1)} & -1 \\
\frac{\gamma(\rho+1)}{(\gamma-1) \rho} & -\frac{\gamma(\rho+1)}{\gamma-1} & 0 \\
\frac{(\gamma-2) \rho-\gamma}{2(\gamma-1) \rho} & \frac{\gamma(\rho+3)-2}{2(\gamma-1)} & 1-\gamma
\end{array}\right]
$$

Dicha matriz presenta siempre un valor propio real negativo, y dos valores dominantes complejos. Mediante el criterio de Routh - Hurwitz es posible determinar que todos sus valores propios poseen parte real negativa y por lo tanto el sistema lineal es siempre estable. Para analizar el efecto de la compartición en los valores propios de la matriz, se obtuvieron las curvas de dichos valores propios para distintos valores de $\gamma>1$, variando el valor de $\rho$ desde $\rho=1$ que corresponde al cliente "igualitario", a $\rho>1$ que modela al cliente elitista.

En la Figura 14 se observa como varían los valores propios complejos de $J$. Cada curva representa un valor de $\gamma$ distinto $\mathrm{y}$ muestra la variación de los valores propios 
al variar $\rho$, aumentando desde $\rho=1$ en la curva negra. Se puede observar que en todos los casos, para un valor de $\gamma$ determinado, al aumentar $\rho$ el sistema se amortigua. En la Figura 15 se muestra el cambio del amortiguamiento para distintos valores de $\gamma$. Esto explica cualitativamente la disminución de las oscilaciones.

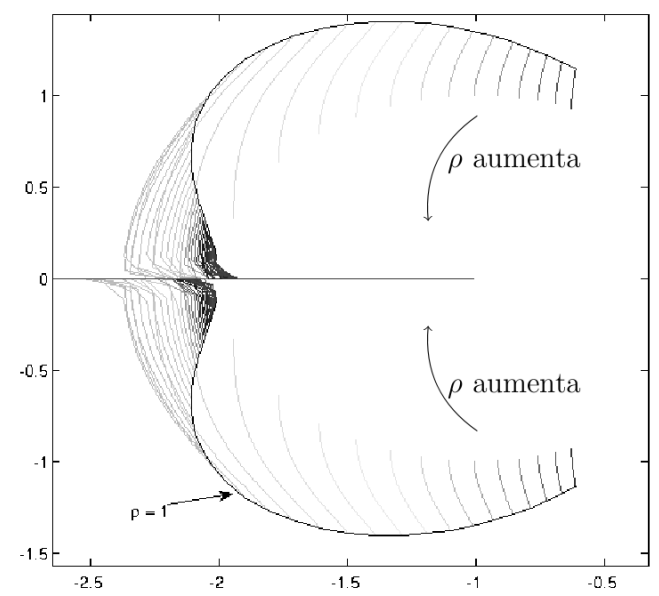

Figura 14 - Lugar de los valores propios complejos de $J$.

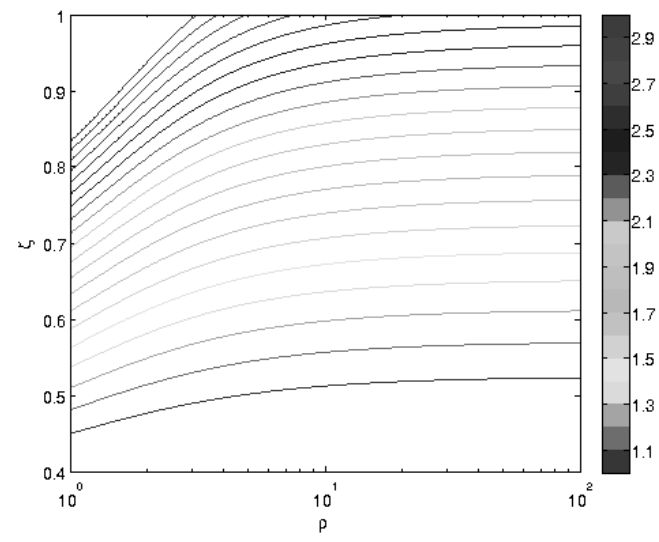

Figura 15 - Amortiguamiento en función de $\rho$ y parametrizado en $\gamma$.

\section{Conclusiones}

En este trabajo se estudió la dinámica del sistema BitTorrent, buscándose validar el modelo de Qiu y Srikant (2004). Para esto se realizaron simulaciones NS-2 de la dinámica en distintas situaciones de los parámetros. Se observó que los valores de equilibrio son los esperados. Además de esto, a partir de las simulaciones se pudo concluir que la eficiencia de la compartición de los peers es prácticamente 1 , es decir no hay oportunidades de intercambio desaprovechadas.

Sin embargo, existen situaciones en las cuales el sistema no alcanza un equilibrio, llegándose a la situación de muerte del torrent. Si se fuerza la supervivencia mediante un seeder permanente, se presenta una oscilación indeseada, que va más allá de la natural aleatoriedad del sistema. Se observan aquí dificultades de los leechers para conseguir sus últimas piezas. A pesar de estos problemas, la eficiencia del sistema continúa siendo 1 .

De forma de solucionar este problema, se planteó una modificación al algoritmo de unchoke original. El BitTorrent Elitista consiste en obligar a todos los peers a destinar sus unchokes a aquellos contactos que poseen la mayor cantidad de chunks y por lo tanto se encuentran más cercanos a completar la descarga. Esto se realizó con la idea de disminuir el tiempo de espera de aquellos leechers que no consiguen su pieza final y de esta manera, generar seeders en forma no tan concentrada en el tiempo.

El cambio propuesto logra solucionar en parte el problema presentado, consiguiendo que el sistema presente menores oscilaciones y disminuyendo la dependencia de los seeders permanentes. Como beneficio extra, se observó que el cambio realizado disminuye la variación entre los tiempos de descarga de los distintos peers, aumentando así la justicia del sistema.

Extendiendo el modelo de Qiu y Srikant (2004) de forma de incluir la modificación realizada, es posible ofrecer una explicación a la disminución de las oscilaciones. Al estudiar la dinámica del modelo, se observa que al destinar mayor cantidad de recursos a aquellos leechers más avanzados en la descarga, el sistema presenta modos de mayor amortiguamiento.

En un futuro trabajo, debería investigarse más a fondo al nuevo cliente elitista para determinar si su efecto siempre es beneficioso. Esto incluiría estudios por simulación o experimentos en situaciones más diversas, no sólo la considerada con 
arribos estacionarios. También, estudiar la posibilidad de coexistencia con clientes de BitTorrent que implementen el algoritmo original.

Otro punto importante a considerar es el tema de incentivos para que los leechers compartan su contenido. El sistema TFT habitual ofrece incentivos de este tipo; la variante propuesta no considera ese aspecto. Sería necesario incluir algún mecanismo que requiera que los leechers colaboren con el sistema para ser considerados para los unchokes.

\section{Agradecimientos}

Este trabajo fue financiado parcialmente por ANII-Uruguay, beca BE_INI_2010_2106 y proyecto FCE2009_1_2158.

\section{Referencias}

COHEN, B.: Incentives build robustness in BitTorrent. In: $1^{\text {st }}$ Workshop on the Economics of Peer-2-Peer Systems. Berkley, 2003.

COHEN B.: BEP 3: The BitTorrent Protocol specification. Feb. 2008.

DE VECIANA G.; YANG X.: Fairness, incentives and performance in peer-to-peer. In: Seeds, 250. p. 300$350,2003$.

EGER, K.; HOßFELD T.; BINZENHÖFER A.; KUNZMANN G.: Efficient simulation of large-scale p2p networks: packet-level vs. flow-level. In: Proceedings of the second workshop on Use of P2P, GRID and agents for the development of content networks. New York, NY, USA: UPGRADE '07, ACM, p. 9-16, 2007.

KOZYNSKI F.: Optimización de redes peer-to-peer. Proyecto de fin de carrera. Universidad ORT Uruguay, 2011. Disponible en: http://ort.edu.uy/fi/publicaciones/mate/publicaciones.html

QIU D.; SANG W.: Global stability of peer-to-peer file sharing systems. Computer Communications 31(2), p. 212-219, 2008.

QIU D.; SRIKANT R.: Modeling and performance análisis of BitTorrent-like peer-to-peer networks. ACM SIGCOMM Computer Communication Review 34(4), p. 367-378, 2004.

Theory.org: BitTorrent protocol specification v1.02. Ago. 2010.

Recebido em maio de 2011

Aprovado para publicação em junho de 2011

Fabián Kozynski

Ingeniero en Telecomunicaciones - Universidad ORT Uruguay - Montevideo - Uruguay. Email:kozynski@ort.edu.uy

Andrés Ferragut

Ingeniero Electrico y Profesor Asociado de la catedra de Teoría de las Telecomunicaciones - Universidad ORT Uruguay - Montevideo - Uruguay. E-mail: ferragut@ort.edu.uy

Fernando Paganini

Catedrático de Teoría de las Telecomunicaciones - Universidad ORT Uruguay - Montevideo - Uruguay. Email: paganini@ort.edu.uy 